\title{
15.
}

\section{Über eine Methode, um von Relationen, welche der Longimetrie angehören, zu entsprechenden Sätzen der Planimetrie zu gelangen.}

\author{
(Vom Herrn Prof. Dr. Möbius zu Leipzig.) \\ (Aus den Berichten der Königlich-Sächsischen Gesellschaft der Wissenschaften von 18j̋.)
}

Unter den drei Theilen, in welche man die Geometrie, der Natur des Raumes gemäfs, getheilt hat, ist die Longimetrie der bei weitem einfachste. Denn hier kommen blofs ein System von Puncten in einer Geraden und Relationen zwischen den gegenseitigen Entfernungen der Puncte in Betracht. Alle diese Relationen aber ergeben sich durch wiederholte Anwendung der einfachsten unter ihnen, welche besagt, dafs, wenn zwei Abschnitte einer Geraden einen Grenzpunct gemein haben, und auf verschiedenen Seiten desselben die beiden andern Grenzpuncte liegen, der zwischen diesen andern begriffene Abschnitt der Summe der zwei ersten Abschnitte gleich ist. Oder allgemeiner, und in Zeichen ausgedrückt: Sind $\boldsymbol{A}, \boldsymbol{B}, \boldsymbol{C}$ drei Puncte einer Geraden, so ist immer, in welcher Ordnung auch die drei Puncte in der Geraden auf einander folgen mögen:

$$
\boldsymbol{A B}+\boldsymbol{B C}=\boldsymbol{A C},
$$

dafern nur auf die durch die Stellung der Buchstaben in den Ausdrücken der Abschnitte bestimmten Richtungen und auf die dadurch bedingten Vorzeichen der Abschnitte gehörige Rücksicht genommen wird.

Ungeachtet dieser grofsen Einfachheit der Longimetrie sind doch bereits mehrere sehr merkwürdige in ihr Gebiet gehörige Untersuchungen geführt worden. Schon in den mathematischen Sammlungen des Pappus (7. Buch) findet sich eine lange Reihe von Sätzen, Relationen zwischen Abschnitten einer Geraden betreffend, und die neueren Geometer haben theils diese Sätze erweitert, theils neue hinzugefügt.

In der letztvergangenen Zeit ist es mir glungen, diesen Sätzen eine noch gröfsere Erweiterung zu geben, indem ich auf eine Methode gekommen 
bin, durch welche zu jedem auf ein System von Puncten in einer Geraden sich beziehenden Satze ein entsprechender Satz für ein System von Puncten in einer Ebene gefunden werden kann. Ich gelangte hierzu durch die Betrachtung, dafs, wenn zwischen mehrern Abschnitten einer Geraden eine Gleichung bestehen soll, und wenn die Grenzpuncte aller Abschnitte bis auf einen unmittelbar gegeben sind, dieser eine Punct durch die Gleichung bestimmt, deshalb aber nicht immer construirbar ist, indem es geschehen kann, dafs sein Abstand von einem der gegebenen Puncte, und somit auch von allen übrigen, von complexer Form, und er selbst folglich in der Geraden imaginär wird. Man weifs aber jetzt, dafs ein solcher Punct zwar nicht in der Geraden, in welcher er eigentlich liegen soll, aber doch in einer durch die Gerade zu legenden Ebene als ein reeller Punct construirt werden kann. Ist nämlich $\boldsymbol{A}$ einer der in der Geraden gegebenen Puncte, $\boldsymbol{P}$ der gesuchte, und findet sich der Abschnitt $\boldsymbol{A P}=x+y /-1$, so ist $\boldsymbol{P}$ derjenige Punct der Ebene, dessen rechtwinklige Coordinaten $=x$ und $y$ in Bezug auf die Gerade, als Axe der Abscissen, und den Punct $\boldsymbol{A}$, als Anfangspunct, sind.

Man kann hierdurch veranlafst werden, gleich von vorn herein die Grenzpuncte aller in der Gleichung vorkommenden Abschnitte als imaginäre Puncte der Geraden und somit als reelle Puncte der Ebene zu betrachten. Die Gleichung selbst wird bei dieser Ansicht eine, oder vielmehr zwei Relationen ausdrücken, welche einer ebenen Figur zukommen und derjenigen Relation entsprechen, welche dieselbe Gleichung ursprünglich in Bezug auf ein System von Puncten in einer Geraden darstellt.

Um diese zwei Relationen für die Ebene zu erhalten, könnte man die Abstände der Grenzpuncte der Abschnitte von einem und demselben Puncte der Geraden, als Anfangspuncte, $=x+y \sqrt{ }-1, x^{\prime}+y^{\prime} \sqrt{ }-1$, u. s. w. setzen, wodurch die Abschnitte selbst, als Differenzen je zweier dieser Abstände gleichfalls von complexer Form würden. Die Substitution dieser complexen Ausdrücke für die Abschnitte in der Gleichung zwischen letztern gäbe alsdann ein Resultat von der Form: $\boldsymbol{X}+\boldsymbol{Y} \sqrt{ }-1=0$, wo $\boldsymbol{X}$, so wie $\boldsymbol{Y}$, eine reelle Function der Coordinaten $x$ und $y, x^{\prime}$ und $y^{\prime}$, u. s. w. der Puncte der ebenen Figur ist. Die zwei Relationen selbst aber würden $\boldsymbol{X}=0$ und $\boldsymbol{Y}=0$ sein. - Indessen kann man einen für die Mehrzahl der hierher gebörenden Relationen ungleich geeigneteren Weg einschlagen, um von der Geraden durch das Gehiet des Imaginüren zu der Ebene zu gelangen. Folgendes ist eine nähere Bezeichnung dieses Weges. 
Sind $\boldsymbol{A}$ und $\boldsymbol{B}$ zwei Puncte einer Ebene, so unterscheide man den reellen und den complexen Werth der Strecke $\boldsymbol{A B}$.

Zur Angabe des reellen Werthes ist zuvor eine Linien-Einheit festzusetzen, und von den zwei Richtungen, nach denen die durch $\boldsymbol{A}$ und $\boldsymbol{B}$ zu legende gerade Linie durchgangen werden kann, zu bestimmen, welche die positive sein soll. Der reelle Werth von $\boldsymbol{A B}$ ist alsdann die Zahl, nach welcher $\boldsymbol{A B}$ von der Linien-Einheit gemessen wird, und diese $Z$ ahl ist positiv oder negativ zu nehmen, jenachdem die Richtuug von $\boldsymbol{A}$ nach $\boldsymbol{B}$ die positive oder die negative Richtung der Linie ist.

Um den complexen Werth der Strecke angeben zu können, mufs nächst den vorigen zwei Stücken eine beliebige Richtung in der Ebene als Normalrichtung, und von dem doppelten Sinne, nach welchem eine Linie in der Ebene gedreht werden kann, der eine als der positive vorher noch festgesetzt werden. Nach diesen Bestimmungen ist der complexe Werth von $\boldsymbol{A B}$ gleich dem reellen Werthe von $\boldsymbol{A B}$, multiplicirt in eine gewisse Function des Winkels der Linie $\boldsymbol{A B}$ mit der Normalrichtung, d. i. des Winkels, um welchen eine Linie, welche die Normalrichtung hat, in positivem Sinne gedreht werden mufs, bis ihre Richtung mit der positiven Richtung der Linie $\boldsymbol{A B}$ identisch wird. Die Winkelfunction aber ist, wenn $\alpha$ den Winkel bezeichnet, die bekannte: $\cos \alpha+\sqrt{ }-1 \cdot \sin \alpha$, die im Folgenden kurz durch $\varphi(\alpha)$ ausgedrückt werde. - Bezeichnen wir daher den reellen Werth der Strecke $\boldsymbol{A B}$ einfach mit $\boldsymbol{A B}$, ihren complexen Werth aber mit $[\boldsymbol{A B}]$, und eine Linie, welche die Normalrichtung hat, mit $x$, so ist

$$
[\boldsymbol{A B}]=\boldsymbol{A B} \cdot \varphi\left(\boldsymbol{x}^{\wedge} \boldsymbol{A B}\right) \text {. }
$$

Hieraus ist zunächst leicht ersichtlich, dafs für je drei beliebige Puncte $\boldsymbol{A}, \boldsymbol{B}, \boldsymbol{C}$ der Ebene

$$
[\boldsymbol{A B}]+[\boldsymbol{B C}]=[\boldsymbol{A C}]
$$

ist. Denn in Folge der Natur der Function $\varphi$ drückt diese Gleichung nichts Anderes aus, als dafs, wenn man die Puncte $\boldsymbol{A}, \boldsymbol{B}, \boldsymbol{C}$ das einemal auf die Linie $x$, das anderemal auf eine in der Ebene auf $x$ perpendiculare Linie rechtwinklig projicirt, in dem einen, wie im andern Falle die Summe der Projectionen von $\boldsymbol{A B}$ und von $\boldsymbol{B C}$ der Projection von $\boldsymbol{A C}$ gleich ist. Zwischen den complexen Werthen der gegenseitigen Abstände irgend dreier Puncte in einer Ebene besteht demnach dieselbe einfache Relation, als wie zwischen den reellen Werthen der Abstände, wenn die drei Puncte in einer Geraden liegen. 
Da nun, wie schon im Eingange bemerkt worden, jede zusammengesetztere Relation zwischen Abschnitten einer Geraden als das Resultat der Verbindung solcher einfachen Gleichungen zwischen den gegenseitigen Abständen dreier Puncte anzusehen ist, und da dieselben einfachen Gleichungen auch zwischen je drei Puncten der Ebene Statt finden, nur dafs hier die complexen Werthe der Abstände statt der dortigen reellen zu setzen sind: so mufs jede identische Gleichung zwischen den gegenseitigen Abständen von Puncten in einer Geraden auch dann noch bestehen, wenn man die Puncte in einer Ebene beliebig liegend annimmt, die Abstände aber nicht mehr in reeller, sondern in complexer Bedeutung gelten läfst. Durch weitere Entwickelung der also aufgefafsten Gleichung, was insbesondere mit Anwendung der bekannten Eigenschaften der Function $\varphi$ :

$$
\varphi(\alpha) \cdot \varphi(\beta)=\varphi(\alpha+\beta) \text { und } \frac{\varphi(\alpha)}{\varphi(\beta)}=\varphi(\alpha-\beta)
$$

zu bewerkstelligen sein wird, reducirt sich die Gleichung, wie im Obigen, auf die Form $\boldsymbol{X}+\boldsymbol{Y} \sqrt{ }-1=0$, und die Gleichungen $\boldsymbol{X}=\mathbf{0}, \boldsymbol{Y}=\mathbf{0}$ liefern die gesuchten Beziehungen.

Nachstehende zwei Beispiele werden den Gegenstand in ein noch helleres Licht setzen. Dabei werde ich zugleich Gelegenheit nehmen, den Gebrauch eines Algorithmus für Winkel zu zeigen, der demjenigen entspricht, dessen ich mich zuerst in meinem „barycentrischen Calcul" für Linien, Dreiecksflächen und Tetraëder bedient habe, eines Algorithmus, der mit Anwendung von Zeichen für Dinge, denen keine Gröfse, blofs Lage zukommt, die arithmetischen Beziehungen zwischen den Theilen der Figur durch Formeln darstellt, welche für alle denkbaren Lagen der Theile Gültigkeit haben. Baryc. Calc. S. XIV.

Reduction eines Vierecks auf ein Dreieck.

Sind $\boldsymbol{A}, \boldsymbol{B}, \boldsymbol{C}, \boldsymbol{D}$ vier beliebige Puncte einer Geraden, so ist

$$
B C=B D-C D, \quad C A=C D-A D, \quad A B=A D-B D .
$$

Multiplicirt man diese drei Gleichungen resp. mit $\boldsymbol{A D}, \boldsymbol{B D}, \boldsymbol{C D}$, und addirt sie hierauf, so kommt

$$
A D \cdot B C+B D \cdot C A+C D \cdot A B=0 .
$$

Es mufs daher auch, wenn vier Puncte $A, \ldots D$ irgendwie in einer Ebene liegen, die Relation bestehen:

$$
[A D][B C]+[B D][C A]+[C D][A B]=0 .
$$


Nun ist

folglich

$$
[\boldsymbol{A D}]=\boldsymbol{A D} \cdot \varphi\left(\boldsymbol{x}^{\wedge} \boldsymbol{A D}\right) \quad \text { und } \quad[\boldsymbol{B C}]=\boldsymbol{B C} \cdot \varphi\left(\boldsymbol{x}^{\wedge} \boldsymbol{B C}\right)
$$

$$
[A D][B C]=A D \cdot B C \cdot \varphi\left(x \wedge D+x^{\wedge} B C\right) ;
$$

und ähnlicher Weise lassen sich die beiden andern Glieder in (1) umformen, setzt man daher der Kürze willen

(2) $\quad \boldsymbol{A D} . \boldsymbol{B C}=\mu, \quad \boldsymbol{B D} \cdot \boldsymbol{C A}=q, \quad \boldsymbol{C D} \cdot \boldsymbol{A B}=\boldsymbol{r} \quad$ und

$$
x^{\wedge} \boldsymbol{A D}+x^{\wedge} \boldsymbol{B C}=\alpha, \quad x^{\wedge} \boldsymbol{B D}+x^{\wedge} \boldsymbol{C A}=\beta, \quad \boldsymbol{x}^{\wedge} \boldsymbol{C D}+x^{\wedge} \boldsymbol{A B}=\gamma,
$$

so verwandelt sich $(1)$ in

$$
\boldsymbol{\mu} \cdot \varphi(\alpha)+\boldsymbol{q} \cdot \varphi(\beta)+\boldsymbol{r} \cdot \varphi(\gamma)=0,
$$

eine Gleichưug, die durch Trennung ihres reellen Theils vom imaginären in die zwei zerfällt:

$$
\left\{\begin{array}{l}
p \cos \alpha+q \cos \beta+r \cos \gamma=0 \\
p \sin \alpha+q \sin \beta+r \sin \gamma=0 .
\end{array}\right.
$$

Seien nun, um dieses Resultat anschaulich auszudrücken, $f, g, h$ drei in einer Ebene liegende und sich nicht in einem Puncte schneidende Gerade, welche mit einer vierten Geraden $v$ der Ebene Winkel bilden, die resp. $=\alpha, \beta, \gamma$ sind. Man nenne die Durchschnitte von $g$ mit $h$, von $h$ mit $f$, von $f$ mit $g$ resp. $\boldsymbol{F}, \boldsymbol{G}, \boldsymbol{H}$, so ist

$$
\boldsymbol{v}^{\wedge} \boldsymbol{G H}=\alpha, \quad \boldsymbol{v}^{\wedge} \boldsymbol{H} \boldsymbol{H}=\beta, \quad \boldsymbol{v}^{\wedge} \boldsymbol{H} \boldsymbol{G}=\gamma
$$

Nächstdem hat man $[\boldsymbol{G H}]+[\boldsymbol{H} \boldsymbol{H}]+[\boldsymbol{F G}]=\mathbf{0}$, d. i., wenn man $\boldsymbol{v}$ zur Normalrichtung nimmt:

$$
\boldsymbol{G H} \cdot \varphi(\alpha)+\boldsymbol{H F} \cdot \varphi(\beta)+\boldsymbol{F} \boldsymbol{G} \cdot \varphi(\gamma)=0,
$$

eine Gleichung, die sich, wie die vorige $p \cdot \varphi(\alpha)+\cdots=0$, in die zwei spaltet:

$$
\begin{aligned}
& \boldsymbol{G H} \cos \alpha+\boldsymbol{H} \boldsymbol{F} \cos \beta+\boldsymbol{H} \boldsymbol{G} \cos \gamma=\mathbf{0}, \\
& \boldsymbol{G H} \sin \alpha+\boldsymbol{H} \boldsymbol{F} \sin \beta+\boldsymbol{F} \boldsymbol{G} \sin \gamma=\mathbf{0} .
\end{aligned}
$$

Hieraus aber und aus (4) folgt $\boldsymbol{G H}: \boldsymbol{H F}: \boldsymbol{F G}=\sin (\gamma-\beta): \sin (\alpha-\gamma)$ : $\sin (\beta-\alpha)=p: q: r$, also

(6) GH:HF: $\mathrm{HG}=A D \cdot B C: B D \cdot C A: C D \cdot A B$.

Ferner ist nach (5) und (3) zu schliefsen:

$$
\boldsymbol{v}^{\wedge} \boldsymbol{F G}-\boldsymbol{v}^{\wedge} \boldsymbol{H F}=\boldsymbol{x}^{\wedge} \boldsymbol{C D}+\boldsymbol{x}^{\wedge} \boldsymbol{A B}-\boldsymbol{x}^{\wedge} \boldsymbol{B D}-\boldsymbol{x}^{\wedge} \boldsymbol{C A} .
$$

Sowie aber allgemein, wenn $a, b, c$ drei in einer Geraden liegende Puncte bedeuten, $a c-a b=b c$ ist, so ist mit gleicher Allgemeinheit, wenn Crelle's Journal f. d. M. Bd. LII. Heft 3. 
$a, b, c$ drei in einer Ebene enthaltene Gerade vorstellen:

$$
\boldsymbol{a}^{\wedge} \boldsymbol{c}-\boldsymbol{a}^{\wedge} \boldsymbol{b}=\boldsymbol{b}^{\wedge} \boldsymbol{c} .
$$

Hiermit reducirt sich die vorige Gleichung auf

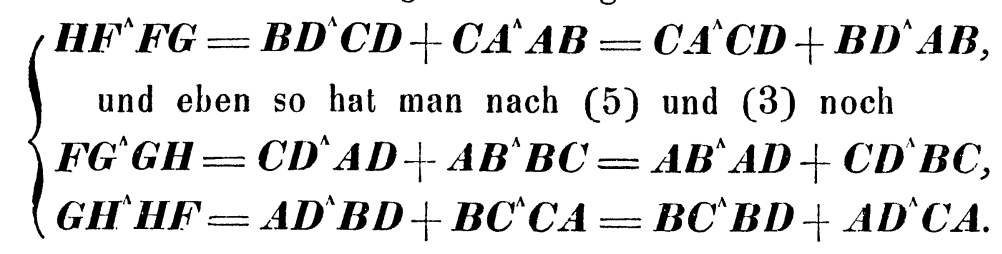

Construirt man daher zu einem Viereck $\boldsymbol{A B C D}$ ein Dreieck $\boldsymbol{F G H}$, dessen Seiten die aus den Winkeln im Viereck durch (7) bestimmten Winkel mit einander machen, so verhalten sich nach (6) die Seiten des Dreiecks wie die Producte $\boldsymbol{p}, q, \boldsymbol{r}$ aus den Seiten und Diagonalen des Vierecks. Es wird daher auch umgekehrt von diesen drei Producten bei einem Viereck ein jedes, absolut genommen, kleiner als die Summe der beiden übrigen sein, so dafs man mit Linien, die ihnen proportional sind, ein Dreieck beschreiben kann; und in diesem Dreieck werden die Winkel die in (7) angegebenen Werthe haben, oder auch, nach dem Sinne, in welchem das Dreieck verzeichnet worden, den Ergänzungen dieser Werthe zu $360^{\prime \prime}$ gleich sein.

Immer aber sind bei den Formeln (6) und (7), wenn sie allgemeine Gültigkeit haben sollen, die positiven Richtungen der in ihnen vorkommenden Linien gehörig zu berücksichtigen. Ursprünglich können die positiven Richtungen der sechs je zwei der vier Puncte $\boldsymbol{A}, \boldsymbol{B}, \boldsymbol{C}, \boldsymbol{D}$ verbindenden Geraden, ingleichen die von einer der Seiten des Dreiecks, es sei von $\boldsymbol{G H}$, nach Belieben gewählt werden. Hiernach bestimmen sich die Vorzeichen der sieben Abschnitte $\boldsymbol{A D}, \boldsymbol{B D}, \ldots \boldsymbol{A B}$ und $\boldsymbol{G H}$, und damit nach (6) die Vorzeichen, also auch die positiven Richtungen von $\boldsymbol{H F}$ und $\boldsymbol{F G}$. Hiermit aber sind, wenn wir noch den positiven Sinn der Drehung in den Ebenen des Vierecks und des Dreiecks festgesetzt baben, die in (7) enthaltenen Winkel vollkommen bestimmt. Es bedeutet nämlich $\boldsymbol{H F}^{\wedge} \boldsymbol{F} \boldsymbol{G}$ den Winkel, um welchen die durch $\boldsymbol{H}$ und $\boldsymbol{F}$ zu legende Gerade in positivem Sinne gedreht werden mufs, bis ihre positive Richtung mit der positiven Richtung der durch $\boldsymbol{F}$ und $\boldsymbol{G}$ zu legenden Geraden einerlei wird; und Analoges gilt von den übrigen WinkelAusdrücken. - Bemerken wir noch, dafs hiernach bei allen den Ausdrücken $\boldsymbol{A D}, \boldsymbol{B D}, \ldots \boldsymbol{F G}$ die Aufeinanderfolge der zwei Buchstaben eines jeden in der Proportion (6) wohl zu beachten ist, nicht mehr aber in den Gleichungen (7). Denn in (6) haben die Strecken $\boldsymbol{G H}$ und $\boldsymbol{H} \boldsymbol{G}$ entgegenge- 
setzte Werthe; dagegen wird in (7) durch $\boldsymbol{G H}$ eben so gut, als durch $\boldsymbol{H} \boldsymbol{G}$, eine durch $\boldsymbol{G}$ und $\boldsymbol{H}$ zu legende Gerade ausgedrückt.

Am einfachsten ist es nun, die positiven Richtungen der sieben Geraden $\boldsymbol{A D}, \boldsymbol{B D}, \ldots \boldsymbol{A B}$ und $\boldsymbol{G H}$ so zu bestimmen, dafs die gleichnamigen Ausdrücke ihrer Abschnitte in (6) positiv werden, also die positive Richtung in $\boldsymbol{A D}$ von $\boldsymbol{A}$ nach $\boldsymbol{D}$ gehend, u. s. w. anzunehmen. Damit werden auch die Abschnitte $\boldsymbol{H F}$ und $\boldsymbol{F} \boldsymbol{G}$ positiv, folglich die Richtungen von $\boldsymbol{H}$ nach $\boldsymbol{F}$ und von $\boldsymbol{F}$ nach $\boldsymbol{G}$ positiv.

Da auf solche Weise in den Winkelgleichungen, wie sie in (7) geschrieben worden, die durch die Aufeinanderfolge der Buchstaben ausgedrückten Richtungen insgesammt positiv genommen werden können, so können wir, den Begriff der positiven Richtung ganz beseitigend, die Winkel-Ausdrücke in diesen Gleichungen auch also deuten, dafs wir $P Q^{\wedge} R S$ als den Winkel nehmen, um welchen die Linie $\boldsymbol{P} \boldsymbol{Q}$ in positivem Sinne gedreht werden mufs, bis ihre durch die Stellung ihrer Buchstaben ausgedrückte Richtung von $\boldsymbol{P}$ nach $\boldsymbol{Q}$ mit der durch die Stellung der Buchstaben der andern Linie ausgedrückten Richtung von $\boldsymbol{R}$ nach $\boldsymbol{S}$ identisch wird.

Hiernach aber sind wir zugleich in den Stand gesetzt, den Winkelgleichungen eine leichter aufzufassende Form zu geben. Berücksichtigen wir nämlich, dafs $P Q^{\wedge} \boldsymbol{Q P}$ nach voriger Erklärung $=180^{\circ}$ ist, und drücken wir den Winkel $\boldsymbol{P} \boldsymbol{Q}^{\wedge} \boldsymbol{P R}$ einfach, und wie es gewöhnlich ist, durch $\boldsymbol{Q P R}$ aus, so wird

$$
\begin{aligned}
& H^{\prime} F^{\prime}=H F^{\wedge} F H+F H F G=180^{\prime \prime}+H F G \text {, } \\
& B D^{\wedge} C D=180^{\prime \prime}+D B^{\wedge} C D=2.180^{\prime \prime}+D B^{\wedge} D C=B D C, \\
& C A^{\wedge} A B=180^{\prime \prime}+A C^{\wedge} A B=180^{\prime \prime}+C A B \text {, u. s. w. }
\end{aligned}
$$

und die Gleichungen (7) reduciren sich damit auf

$$
\left\{\begin{array}{l}
H F G=B D C+C A B=A C D+D B A, \\
F G H=C D A+A B C=B A D+D C B, \\
G H F=A D B+B C A=C B D+D A C
\end{array}\right.
$$

wofür wir auch, weil $C A B+B A C=A C^{\wedge} A B+A B^{\wedge} A C=A C^{\wedge} A C=0$, u. s. w. ist, schreiben können:

$$
\left\{\begin{array}{l}
H H G=B D C-B A C=D B A-D C A \\
F G H=C D A-C B A=D C B-D A B \\
G H F=A D B-A C B=D A C-D B C
\end{array}\right.
$$


Während wir also die Aufeinanderfolge der Buchstaben bei deren paarweisen Verbindungen anfänglich nur in der Gleichung (6) zwischen $\mathbf{A b -}$ schnitten, nicht mehr aber in den Winkelgleichungen (7) zu beachten hatten, kommt jetzt, umgekehrt, die Stellung der Buchstaben blofs bei letztern Gleichungen in Betracht und kann bei erstern ganz vernachlässigt werden, indem es bei der Construction des Dreiecks FGH mittels der Seiten desselben blofs auf die absoluten Werthe der Producte $p, q, r$ ankommt, denen die Seiten proportional sein sollen; und wir sind somit, ohne dafs die allgemeine Anwendbarkeit der Gleichungen in Etwas beeinträchtigt würde, einer vorläufigen Bestimmung der positiven Richtungen der Linien ganz überhoben.

Übrigens müssen die Gleichungen (8) und $\left(8^{*}\right)$, die wir unter der Hypothese entwickelten, dafs die sieben durch $\boldsymbol{A D}, \ldots \boldsymbol{A B}$ und $\boldsymbol{G H}$ ausgedrückten Richtungen insgesammt positiv seien, auch unter jeder andern $\mathrm{Hy}-$ pothese über diese Richtungen sich wieder finden. Nehmen wir z. B. von jenen sieben Richtungen $\boldsymbol{C A}$ negativ an und lassen die sechs übrigen positiv, so wird nach (6) $\boldsymbol{H F}$ negativ, $\boldsymbol{F G}$ aber bleibt positiv. Damit ist in den Gleichungen (7), um die zuletzt gegebene Definition eines Winkel-Ausdrucks auf sie anwendbar zu machen, $\boldsymbol{A C}$ und $\boldsymbol{F H}$ statt $\boldsymbol{C A}$ und $\boldsymbol{H F}$ zu schreiben. Die erste und die dritte derselben, denn nur hierin kommen diese Richtungen vor, verwandeln sich dadurch in

$$
\begin{aligned}
& F H^{\wedge} \boldsymbol{H} G=B D^{\wedge} \boldsymbol{C D}+\boldsymbol{A} \boldsymbol{C}^{\wedge} \boldsymbol{A B}=\cdots, \\
& G H^{\wedge} \boldsymbol{F H}=\boldsymbol{A} \boldsymbol{D}^{\wedge} \boldsymbol{B D}+\boldsymbol{B} \boldsymbol{C}^{\wedge} \boldsymbol{A C}=\cdots,
\end{aligned}
$$

und man sieht von selbst, wie diese Gleichungen mit den entsprechenden in (8) übeinstimmen.

Das Resultat endlich, das durch (6) und (8) oder (8*) ausgedrückt wird, liefse sich etwa also in Worte fassen:

Hat man in einer Ebene vier Puncte $(A, B, C, D)$ und multiplicirt den gegenseitigen Abstand je zweier derselben mit dem gegenseitigen Abslande der jedesmal zwei übriyen, so kann man mit Linien, welche den drei erhallenen Producten proportional sind, ein Dreieck (HGH) construiren. Jeder Winkel (wie F) dieses Dreiecks aber ist dem Unlerschiede der Winkel gleich, unter welchen von den zwei Abstünden ( $\boldsymbol{D A}$ und $\boldsymbol{B C}$ ), deren Product der dem Winkel gegenüberliegenden Dreiecksseite $(\boldsymbol{G H})$ proportional ist, der eine Abstand von den Endpuncten 
des andern aus ( $\boldsymbol{B C}$ von $\boldsymbol{D}$ und $\boldsymbol{A}$ aus oder, welches gleichviel ist, $\boldsymbol{D} \boldsymbol{A}$ von $\boldsymbol{B}$ und $\boldsymbol{C}$ aus betrachtet) erscheint.

Hieran knüpft sich unmittelbar die merkwürdige Folgerung: Sind bei einem ebenen Viereck von den drei Verhältnissen zwischen den gedachten drei Producten und von den gedachten drei Winkeldifferensen, sind von diesen sechs Stücken irgend zwei gegeben, so kann man daraus die vier übrigen finden, - ganz eben so, wie man bei einem Dreieck aus irgend zweien der sechs Stücke, nämlich der drei Verhältnisse zwischen den Seiten und der drei Winkel, die vier übrigen bestimmen kann.

Eine besondere Erwähnung dürften noch die nachstehenden zwei speciellen Fälle verdienen.

1. Sind drei der vier Puncte $\boldsymbol{A}, \ldots \boldsymbol{D}$, etwa $\boldsymbol{A}, \boldsymbol{B}, \boldsymbol{C}$ gleichweit von einander entfernt, ist also $\boldsymbol{D}$ irgend ein Punct in der Ebene eines gleichseitigen Dreiecks $\boldsymbol{A B C}$, so verhalten sich die Producte $p, q, r$ wie $\boldsymbol{A D}$, $\boldsymbol{B D}, \boldsymbol{C D}$. Man kann folglich alsdann mit $\boldsymbol{A D}, \boldsymbol{B D}, \boldsymbol{C D}$ selbst ein Dreieck construiren, und die Winkel dieses Dreiecks werden resp. $=\boldsymbol{B D C}-\boldsymbol{B} \boldsymbol{A C}$, u. s. w. sein.

2. Sind $\boldsymbol{P}, \boldsymbol{Q}, \boldsymbol{R}, \boldsymbol{S}$ vier Puncte eines Kreises, so ist, jenachdem $\boldsymbol{R}$ und $\boldsymbol{S}$ auf einerlei, oder verschiedenen Seiten der Geraden $\boldsymbol{P} \boldsymbol{Q}$ liegen, der Winkel-Unterschied PRQ-PSQ entweder $=0$, oder $=180^{\prime \prime}$, vorausgesetzt immer, dafs alle in derselben Ebene enthaltenen Winkel nach einerlei Sinne gerechnet werden.

Liegen daher die vier Puncte $\boldsymbol{A}, \boldsymbol{B}, \boldsymbol{C}, \boldsymbol{D}$ in einem Kreise, und zwar $\boldsymbol{C}$ und $\boldsymbol{D}$ auf verschiedenen Seiten von $\boldsymbol{A B}$, so ist $\boldsymbol{B D C}-\boldsymbol{B} \boldsymbol{A C}=\mathbf{0}$, $\boldsymbol{C D} \boldsymbol{A}-\boldsymbol{C B} \boldsymbol{A}=0, \boldsymbol{A D B}-\boldsymbol{A C B}=180^{\prime \prime} ;$ folglich nach $\left(8^{*}\right)$
1) $\boldsymbol{H F G}=0$
2) $\boldsymbol{F} \boldsymbol{G H}=0$,
3) $\boldsymbol{G H}=180^{\prime \prime}$.

Wegen 1) haben $\boldsymbol{F H}$ und $\boldsymbol{F} \boldsymbol{G}$ einerlei Richtung, desgleichen $\boldsymbol{G} \boldsymbol{F}$ und $\boldsymbol{G H}$ wegen 2). Mithin, und wie auch schon aus 3 ) allein folgt, liegen jetzt $\boldsymbol{F}$, $\boldsymbol{G}, \boldsymbol{H}$ in gerader Linie, $\boldsymbol{H}$ zwischen $\boldsymbol{F}$ und $\boldsymbol{G}$; und es ist daher in absolutem Sinne, d. h. abgesehen von der durch die Stellung der Buchstaben angedeuteten Richtung: $\boldsymbol{G H}+\boldsymbol{H} \boldsymbol{H}=\boldsymbol{F G}$. Hieraus aber folgt nach (6) die allbekannte Gleichung zwischen den Seiten und Diagonalen eines in einen Kreis beschriebenen Vierecks:

$$
\boldsymbol{A D} \cdot \boldsymbol{B C}+\boldsymbol{B D} \cdot \boldsymbol{C A}=\boldsymbol{C D} \cdot \boldsymbol{A B} .
$$


Harmonische Lage von vier Puncten in einer Ebene.

Wenn wir in der zu Anfange des vorigen Artikels aufgestellten zwischen je vier Puncten einer Geraden gültigen Gleichung zwei der drei Producte, deren Summe null war, etwa die zwei ersten, einander gleich setzen, also

(1) $\boldsymbol{A D} \cdot \boldsymbol{B C}=\boldsymbol{B D} \cdot \boldsymbol{C A}$

annehmen, so reducirt sich jene Gleichung auf

(2) $2 \boldsymbol{B D} . \boldsymbol{C A}=\boldsymbol{B A} . \boldsymbol{C D}$.

Bezeichnet ferner $\boldsymbol{M}$ den Mittelpunct von $\boldsymbol{A B}$, also einen dergestalt in der Geraden liegenden Punct, dafs

(3) $\quad \boldsymbol{M A}+\boldsymbol{M B}=\mathbf{0}$

ist, so hat man

$$
\begin{aligned}
& \boldsymbol{A D}=\boldsymbol{M D}-\boldsymbol{M A}, \boldsymbol{B C}=\boldsymbol{M C}-\boldsymbol{M B}=\boldsymbol{M C}+\boldsymbol{M A}, \\
& \boldsymbol{B D}=\boldsymbol{M D}-\boldsymbol{M B}=\boldsymbol{M D}+\boldsymbol{M A}, \boldsymbol{C A}=\boldsymbol{M A}-\boldsymbol{M C} .
\end{aligned}
$$

Mit diesen Werthen für $\boldsymbol{A D}, \boldsymbol{B C}$, u. s. w. geht (1) über in

(4) $\quad M A^{2}=M C . M D$.

Eine andere Form, die man der Gleichung (1) geben kann, ist

$$
(\boldsymbol{C A}-\boldsymbol{C D}) \boldsymbol{C B}=(\boldsymbol{C D}-\boldsymbol{C B}) \boldsymbol{C A},
$$

d. i.

$$
2 \boldsymbol{C A} \cdot \boldsymbol{C B}=\boldsymbol{C D}(\boldsymbol{C A}+\boldsymbol{C B}),
$$

oder, weil $\boldsymbol{C A}+\boldsymbol{C B}=\boldsymbol{C M}+\boldsymbol{M A}+\boldsymbol{C M}+\boldsymbol{M B}=\mathbf{2} \boldsymbol{C M}$ ist,

$$
\text { (5) } \boldsymbol{C A} \cdot \mathrm{CB}=\mathrm{CD} . \mathrm{CM} \text {; }
$$

und solcher Umwandlungen von (1) liefsen sich noch verschiedene andere bewerkstelligen.

Wenden wir auf die jetzt gemachten die im Obigen erörterte Methode an, so wird, wenn man zu drei nicht in einer Geraden liegenden Puncten $\boldsymbol{A}, \boldsymbol{B}, \boldsymbol{C}$ einen in ihrer Ebene begriffenen Punct $\boldsymbol{D}$ so bestimmt, dafs

$$
\text { [1] }[A D][B C]=[B D][C A]
$$

ist, - so wird dann auch

$$
\text { [2] } 2[\boldsymbol{B D}][\boldsymbol{C A}]=[\boldsymbol{B A}][\boldsymbol{C D}]
$$


sein; und wenn man einen Punct $M$ hinzufügt, so dafs

[3] $[\boldsymbol{M A}]+[\boldsymbol{M B}]=0$,

so wird man noch

[4] $[\boldsymbol{M A}]^{2}=\left[M C^{\prime}\right][M D]$

und

[5] $[\boldsymbol{C A}][\boldsymbol{C B}]=[\boldsymbol{C D}][\boldsymbol{C M}]$ haben.

Nun wird durch die Gleichung (1), die man auch in Form der Proportion

$$
\boldsymbol{C A}: \boldsymbol{C B}=-\boldsymbol{D A}: \boldsymbol{D B}
$$

schreiben kann, ausgedrückt, dafs die Linie $\boldsymbol{A B}$ in $\boldsymbol{C}$ und $\boldsymbol{D}$ nach Verhältnissen getheilt wird, deren Exponenten einander gleich und entgegengesetzt sind. Bekanntlich ist dieses die Definition der harmonischen Theilung von $\boldsymbol{A B}$ in $\boldsymbol{C}$ und $\boldsymbol{D}$, sowie von $\boldsymbol{C D}$ in $\boldsymbol{A}$ und $\boldsymbol{B}$. Man wird daher auch sagen können, dafs zwei in einer Ebene enthaltene Paare von Puncten, $\boldsymbol{A}$ und $\boldsymbol{B}$, $\boldsymbol{C}$ und $\boldsymbol{D}$, eine harmonische Lage gegen einander haben, wenn zwischen den complexen Werthen der Abstände der Puncte des einen Paares von denen des andern die Gleichung [1] besteht. Und so wie die aus (1) fliefsenden Gleichungen (2), (4), (5) noch andere Eigenschaften zweier in einer Geraden harmonisch liegenden Paare von Puncten ausdrücken, so werden durch [2], [4], [5] die entsprechenden Eigenschaften zweier harmonischen Paare in einer Ebene dargestellt werden.

Es ist jetzt noch übrig die reelle Bedeutung von [1] .. [5] zu entwickeln. - Die Gleichung [1] ist identisch mit

Es ist aber

$$
\text { (a) } \frac{[C B][D A]}{[C A][D B]}=-1 \text {. }
$$

$$
\begin{aligned}
& \frac{[C B]}{[C A]}=\frac{C B \cdot \varphi\left(x^{\wedge} \boldsymbol{C B}\right)}{\boldsymbol{C A} \cdot \varphi\left(x^{\wedge} \boldsymbol{C A}\right)}=\frac{\boldsymbol{C B}}{\boldsymbol{C A}} \cdot \varphi(\boldsymbol{C A} \boldsymbol{C} B), \text { und eben so } \\
& \frac{[\boldsymbol{D A}]}{[\boldsymbol{D B}]}=\frac{\boldsymbol{D A}}{\boldsymbol{D B}} \varphi\left(\boldsymbol{D B} \boldsymbol{B}^{\wedge} \boldsymbol{D} \boldsymbol{A}\right) \text {. Damit verwandelt sich }(a) \text { in } \\
& \text { (b) } \frac{C B}{C A} \cdot \frac{D A}{D B} \varphi(\alpha)=-1, \text { wo } \\
& \boldsymbol{a}=\boldsymbol{C} \boldsymbol{A}^{\wedge} \boldsymbol{C B}+\boldsymbol{D B} \boldsymbol{B}^{\wedge} \boldsymbol{D A}=\boldsymbol{C} \boldsymbol{A}^{\wedge} \boldsymbol{C B}-D A^{\wedge} D B \text {. }
\end{aligned}
$$

Die Gleichung (b) kann aber nicht anders bestehen, als wenn $\varphi \alpha$ einen reellen Werth hat, also entweder $=1$ oder -1 , und damit $\alpha$ entweder $=0$, oder $=180^{\prime \prime}$ ist. Beide Hypothesen führen zu demselben Resultate. 
Nehmen wir die letztere an, so wird

(c) $\boldsymbol{C B} \cdot \boldsymbol{D A}=\boldsymbol{C A} \cdot \boldsymbol{D B}$

In Folge von (c) können wir die Richtungen von $\boldsymbol{C A}, \boldsymbol{C B}, \boldsymbol{D A}, \boldsymbol{D B}$ sämmtlich positiv sein lassen und alsdann, den im vorigen Artikel gegebenen Erörterungen gemäfs, statt $(d)$ auch schreiben:

$$
\text { (e) } \quad A C B-A D B=180^{\prime \prime} \text {. }
$$

Die harmonische Lage der Punclenpaare $A$ und $B, C$ und $D$ in einer Ebene wird hiernach durch die zwei Gleichungen $(c)$ und $(e)$ bedingt. Wegen (e) müssen die vier Puncte in einem Kreise liegen, und zwar $C$ und $D$ auf verschiedenen Seiten von $A B$, also in der Folge $A, C$, $\boldsymbol{B}, \boldsymbol{D}$. Wegen (c) inüssen die zwei Producte der gegenüberliegenden Seiten des Vierecks $A C B D$ einander gleich sein. Auch kann statt (c) die leicht aufzufassende Proportion

$$
\boldsymbol{C A}: \boldsymbol{C B}=\boldsymbol{D A}: \boldsymbol{D B} \text { oder } \quad \boldsymbol{A C}: \boldsymbol{A D}=\boldsymbol{B C}: \boldsymbol{B D}
$$

gesetzt werden.

Was nun die übrigen Eigenschaften der harmonischen Lage betrifft, so folgt zunächst aus der Gleichung [2], wenn man sie ähnlicherweise wie [1] behandelt, aufser der Gleichheit der Winkel $\boldsymbol{A B D}$ und $\boldsymbol{A C D}$ und der daraus fliefsenden Kreislage der vier Puncte, dafs die Gleichung (2) auch für die Ebene gilt, und dafs somit das Product aus den Diagonalen des Vierecks $\boldsymbol{A C B D}$ doppelt so grofs als das Product des einen oder des andern Paars gegenüberliegender Seilen ist, - was auch unmittelbar aus der eben aufgestellten Definition der harmonischen Lage in Verbindung mit dem Satze am Ende des vorigen Artikels hervorgeht.

Aus [3] ersieht man leicht, dafs $\boldsymbol{M}$ auch jetzt noch der Mittelpunct der Linie $\boldsymbol{A B}$ ist. - Die Gleichung [4] wird nach dem bei [1] gezeigten Verfahren:

$$
\frac{M A}{M C} \cdot \frac{M A}{M D} \varphi(\alpha)=1, \quad \text { wo } \alpha=M C^{\wedge} M A+M D^{\wedge} M A \text {. }
$$

Nehmen wir hierin die Richtungen $\boldsymbol{M A}, \boldsymbol{M C}, \boldsymbol{M D}$ positiv, so mufs $\varphi(\alpha)=1$, also $\alpha=0$ sein. Dies giebt die Winkelgleichung $\boldsymbol{C M} \boldsymbol{A}=\boldsymbol{A M D}$ und die Proportion $\boldsymbol{M C}: \boldsymbol{M A}=\boldsymbol{M A}: \boldsymbol{M D}$. Aus beiden in Verbindung folgt die Ähnlichkeit und ähnliche Lage der Dreiecke $\boldsymbol{C M} \boldsymbol{A}$ und $\boldsymbol{A M D}$; und dasselbe mufs auch von den Dreiecken $\boldsymbol{C M B}$ und $\boldsymbol{B M D}$ gelten. 
Auf gleiche Weise folgt endlich aus [5], dafs auch die Dreiecke $\boldsymbol{C M B}$ und $\boldsymbol{C A D}$, sowie $\boldsymbol{C M} \boldsymbol{A}$ und $\boldsymbol{C B D}$ einander ähnlich und in ähnlicher Lage sind.

Bei zuei in einer Ebene harmonisch liegenden Paaren von Puncten $\boldsymbol{A}$ und $\boldsymbol{B}, \boldsymbol{C}$ und $\boldsymbol{D}$ sind demnach, wenn $M$ den Mittelpunct des eimen Paares $A B$ bezeichnet,

$$
\begin{aligned}
& \text { die Dreiecke } C M A, A M D, C B D \text {, ingleichen } \\
& \text { die Dreiecke } C M B, B M D, C A D
\end{aligned}
$$

einander ähnlich und in ähnlicher Lage.

Noch läfst sich aus der Gleichheit der Winkel $\boldsymbol{C M A}$ und $\boldsymbol{A M D}$ eine sehr einfache Construction folgern, um, wenn von zwei harmonischen Paaren von Puncten das eine Paar $\boldsymbol{A}$ und $\boldsymbol{B}$, und der eine Punct $\boldsymbol{C}$ des andern Paares gegeben sind, den andern Punct $\boldsymbol{D}$ des letztern zu finden. Man beschreibe nämlich durch $\boldsymbol{A}, \boldsymbol{B}, \boldsymbol{C}$ einen Kreis, trage auf diesen von $\boldsymbol{B}$ aus einen Bogen $\boldsymbol{B E}$, gleich und in gleichem Sinne mit dem Bogen $\boldsymbol{C A}$, und lege durch $\boldsymbol{E}$ und den Mitlelpunct $\boldsymbol{M}$ der Linie $\boldsymbol{A B}$ eine Gerade, so wird der zweite Durchschnitt derselben mit dem Kreise der gesuchte Punct $\boldsymbol{D}$ sein.

Man kann den Punct $\boldsymbol{D}$ auch dadurch finden, dafs man in $\boldsymbol{A}$ und $\boldsymbol{B}$ an den Kreis zwei Tangenten legt und den gegenseitigen Durchschnitt derselben, welcher $\boldsymbol{F}$ heifse, mit $\boldsymbol{C}$ durch eine Gerade verbindet; denn diese wird den Kreis zum zweitenmale in $\boldsymbol{D}$ schneiden. - In der That sind nach dieser Construction die Dreiecke $A F C$ und $D F A$, sowie die Dreiecke $B F C$ und $\boldsymbol{D F B}$ einander ähnlich; mithin

$$
A C: D A=F C: F A \text { und } B C: D B=F C: H B,
$$

woraus, wegen $\boldsymbol{F A}=\boldsymbol{F B}$, die Fundamentalproportion

$$
A C: D A=B C: D B
$$

folgt.

Je zwei Puncte $\boldsymbol{C}$ und $\boldsymbol{D}$ eines Kreises, so schliefsen wir hieraus noch, welche mit zwei bestimmten Puncten $A$ und $B$ des Kreises in Harmonie sind, liegen demnach mit einem bestimmten Puncte $F$ in gerader Linie. - Rückt $C$ unendlich nahe an $\boldsymbol{A}$ oder an $\boldsymbol{B}$, so thut dasselbe, in Folge der Fundamentalproportion, auch $D$, und es mufs daher, wie wir bereits wissen, auch jede der beiden an $\boldsymbol{A}$ und $\boldsymbol{B}$ gelegten Tangenten den Punct $\boldsymbol{F}$ treffen. 
Auf ähnliche Art, wie die harmonische Theilung einer Linie, habe ich auch dic Eigenschaften der Involution von sechs Puncten in einer Geraden auf die Ebene überzutragen gesucht. Die sehr merkwürdigen Resultate, die ich hierbei gefunden, so wie eine neue Art von Verwandtschaft zwischen ebenen Figuren, eine Verwandtschaft, die sich mir durch Übertragung collinear verwandter Systeme von Puncten in einer Geraden auf die Ebene ergab, gedenke ich später mitzutheilen und will hier nur noch bemerken, dafs aus dieser neuen Verwandtschaft eben so, wie aus jeder der schon bekannten, eine besondere Classe von Aufgaben entspringt, und dafs die einfachste dieser Aufgaben die bereits im vorigen Artikel erwähnte ist, wonach bei einem Viereck aus irgend zweien gewisser sechs Stücke jedes der vier übrigen gefunden werden kann. 